\title{
Administration of Eculizumab, a C5 Inhibitor, for the Treatment of Shiga-Toxin-Producing Escherichia coli Infection: A Case Report
}

\author{
Takahiro Yukawa*, Takuto Ishida, Toshinobu Yamagishi, Kazuhiro Sugiyama, Yuichi Hamabe \\ Department of Trauma and Critical Care Center, Tokyo Metropolitan Bokutoh Hospital, Tokyo, Japan \\ Email address: \\ tetr0901@yahoo.co.jp (T. Yukawa) \\ ${ }^{*}$ Corresponding author

\section{To cite this article:} \\ Takahiro Yukawa, Takuto Ishida, Toshinobu Yamagishi, Kazuhiro Sugiyama, Yuichi Hamabe. Administration of Eculizumab, a C5 Inhibitor, \\ for the Treatment of Shiga-Toxin-Producing Escherichia coli Infection: A Case Report. American Journal of Clinical and Experimental \\ Medicine. Vol. 6, No. 1, 2018, pp. 27-32. doi: 10.11648/j.ajcem.20180601.15
}

Received: January 12, 2018; Accepted: February 1, 2018; Published: March 8, 2018

\begin{abstract}
Hemolytic-uremic syndrome (HUS), which is characterized by microvascular hemolytic anemia, consumption thrombocytopenia, and acute renal failure, is a complication of the Shiga-toxin-producing Escherichia coli (STEC) infection. We describe the case of a patient who, despite undergoing plasma exchange and renal replacement therapy for STEC-induced HUS, experienced poor improvement in platelet count, serum creatinine level, and serum lactate dehydrogenase level. The patient developed acute encephalopathy but recovered without permanent organ damage after eculizumab therapy. For severe HUS cases involving the central nervous system, early administration of eculizumab, which inhibits the abnormal activation of the complement activation pathway, may be effective.
\end{abstract}

Keywords: Hemolytic-Uremic Syndrome, Shiga-Toxin-Producing Escherichia coli Infection, Acute Encephalopathy, Eculizumab

\section{Introduction}

The Shiga-toxin-producing Escherichia coli (STEC) is an infectious pathogen that has caused several major disease outbreaks worldwide, including the 1982 outbreak caused by the serotype O157:H7 in the United States [1] and the 2011 outbreak caused by the serotype O104:H4 in Europe, which affected Germany most specifically [2]. In Japan, a case of mass food poisoning caused by the STEC serotype O111 was reported in the Toyama Prefecture in 2011, which resulted in deaths due to the severity of the infection. Hemolytic-uremic syndrome (HUS), which is characterized by microvascular hemolytic anemia, consumption thrombocytopenia, and acute renal failure, is a complication of STEC infection that develops in $6-9 \%$ of STEC infection cases, with a higher specific prevalence of $15 \%$ among children under the age of 10 years [3].

The terminal complement inhibitor, eculizumab, the production of $\mathrm{C} 5 \mathrm{a}$ and the membrane attack complex (MAC) by selectively binding to the complement $\mathrm{C} 5$ protein and inhibiting the complement activation pathway. This results in the suppression of events related to complement-mediated thrombotic microangiopathy (TMA) [4], one of the key characteristics of HUS. In Japan, eculizumab has been approved for the treatment of paroxysmal nocturnal hemoglobinuria $(\mathrm{PNH})$, with its indications having been expanded to the treatment of atypical HUS (aHUS) since 2013. However, to date, there is no disease-specific treatment strategy for STEC-HUS, with supportive treatment generally being recommended, although a few studies have reported on the possible usefulness of eculizumab [5].

In this case report, we describe the clinical course of a patient with STEC-HUS who showed poor improvement in clinical symptoms despite supportive therapy and progression of STEC-HUS to involve the central nervous system. Consequently, we opted to begin treatment using eculizumab, with the patient recovering fully without any permanent damage. To our knowledge, this is the only case report regarding the use of eculizumab for the treatment of 


\section{STEC-HUS in an adult.}

\section{Case Report}

\subsection{Clinical Course of the Illness}

A 16-year-old girl sought treatment at a local clinic after experiencing abdominal pain and bloody diarrhea a day after eating seafood. The patient had an unremarkable past medical history. A fecal culture test was performed and the patient was sent home after being administered an intestinal regulator. As her abdominal pain increased despite treatment, the patient sought further care at a general hospital. An abdominal contrast-enhanced computed tomography (CT) revealed edematous thickening of the pancreatic mucosa, with abdominal ascites. Based on these findings, the patient was urgently admitted with a possible diagnosis of severe bacterial colitis. The fecal culture and direct Shiga-toxin detection test performed at the previous clinic revealed no abnormalities. On Day 5 after developing symptoms, a decreased platelet count, an elevated serum lactate dehydrogenase (LDH) level, and acute renal dysfunction were noted. As HUS was suspected, the patient was transferred to our emergency department for detailed examination and treatment.

\subsection{Presentation upon Admission}

Upon admission, the patient was conscious and alert, reporting intense abdominal pain. No neurological abnormalities were identified on clinical examination. Physiological measures were as follows: blood pressure, 125/76 mmHg; heart rate, 107 beats/min; respiratory rate, 22 breaths $/ \mathrm{min} ; \mathrm{SpO}_{2}, 98 \%$ (at room air); and body temperature, $36.7^{\circ} \mathrm{C}$. Respiration was clear, with no abnormalities on auscultation. The abdomen was generally distended, with tenderness and pain on palpation of the lower abdomen along the midline. There was no pain on rectal examination, with no evidence of hepatosplenomegaly.

Laboratory findings are reported in Table 1, with relevant findings summarized as follows: the platelet count was low, with signs of crushed erythrocytes, hypoalbuminemia, elevated LDH, renal dysfunction, and fibrinolytic coagulation abnormalities.

Table 1. Serum laboratory result on admission.

\begin{tabular}{|c|c|c|c|}
\hline White Blood Cell Count $(/ \mu \mathrm{l})$ & 22,100 & Creatine Kinase (U/I) & 222 \\
\hline Neutrophil (\%) & 82.1 & Creatine Kinase - MB (U/1) & $<3$ \\
\hline Lymphocyte (\%) & 5.8 & Aspartate Aminotransferase (U/l) & 59 \\
\hline Monocyte (\%) & 10.6 & Alanine Aminotransferase (U/l) & 13 \\
\hline Eosinophil (\%) & 0.1 & Lactate Dehydrogenase (U/l) & 1753 \\
\hline Basophil (\%) & 1.4 & Alkaline Phosphatase (U/l) & 145 \\
\hline Hemoglobin (g/dl) & 10.2 & Cholinesterase (U/1) & 63 \\
\hline Hematocrit $(\%)$ & 30.3 & Amylase (U/1) & 129 \\
\hline \multirow[t]{2}{*}{ Platelet $(\times 10,000 / \mu \mathrm{l})$} & 2.6 & Haptoglobin $(\mathrm{mg} / \mathrm{dl})$ & 9 \\
\hline & & Thrombin - Antithrombin Complex (ng/ml) & 13.7 \\
\hline Prothrombin Time (\%) & 53.5 & Alpha-2 Plasmin Inhibitor Complex $(\mu \mathrm{g} / \mathrm{ml})$ & 3.4 \\
\hline International Normalized Ratio of Prothrombin Time & 1.43 & Protein-C activity $(\%)$ & 63 \\
\hline Activated Partial Thromboplastin Time (s) & 34.7 & Protein-S activity (\%) & 32.1 \\
\hline Fibrinogen $(\mathrm{mg} / \mathrm{dl})$ & 548 & ADAMTS-13 activity (\%) & 39.1 \\
\hline D-dimer $(\mu \mathrm{g} / \mathrm{ml})$ & 31.9 & Immunoglobulin G (mg/dl) & 289 \\
\hline Total Protein $(\mathrm{g} / \mathrm{dl})$ & 3.6 & Hemolytic Complement activity $(\mathrm{U} / \mathrm{ml})$ & 49 \\
\hline Albumin $(\mathrm{g} / \mathrm{dl})$ & 1.7 & $\mathrm{C} 3$ complement $(\mathrm{mg} / \mathrm{dl})$ & 61 \\
\hline Blood Urea Nitrogen (mg/dl) & 24.8 & C4 complement (mg/dl) & 17 \\
\hline Creatinine $(\mathrm{mg} / \mathrm{dl})$ & 1.32 & Anti-nuclear Antibody & negative \\
\hline Uric Acid (mg/dl) & 3.0 & Anti-neutrophil Cytoplasmic Antibody & negative \\
\hline Ammonia $(\mu \mathrm{g} / \mathrm{dl})$ & 47 & Direct/indirect Coombs' test & negative \\
\hline Total Bilirubin (mg/dl) & 1.27 & Blood Gas Analysis (6 L/min $\mathrm{O}_{2}$ given) & \\
\hline Sodium $(\mathrm{meq} / \mathrm{l})$ & 129 & $\mathrm{pH}$ & 7.376 \\
\hline Potassium (meq/l) & 4.3 & $\mathrm{PaO}_{2}$ (torr) & 293 \\
\hline Chlorine (meq/l) & 97 & $\mathrm{PaCO}_{2}$ (torr) & 43.4 \\
\hline Calcium (mg/dl) & 7.1 & Hydrogen-carbonate ion (mmol/1) & 24.8 \\
\hline Inorganic Phosphate (mg/dl) & 3.1 & Anion Gap & 3.1 \\
\hline Magnesium (mg/dl) & 1.8 & Lactate $(\mathrm{mmol} / \mathrm{l})$ & 0.6 \\
\hline
\end{tabular}

A 12-lead electrocardiogram was negative, and no abnormal findings were noted on CT of the head. The abdominal contrast-enhanced CT showed extensive edematous thickening of the colon wall and ascites (Figure
1A). The fecal culture test performed at the patient's first visit to a local clinic did not reveal significant phlogogenic fungi, and result of the direct Shiga-toxin detection was negative. 

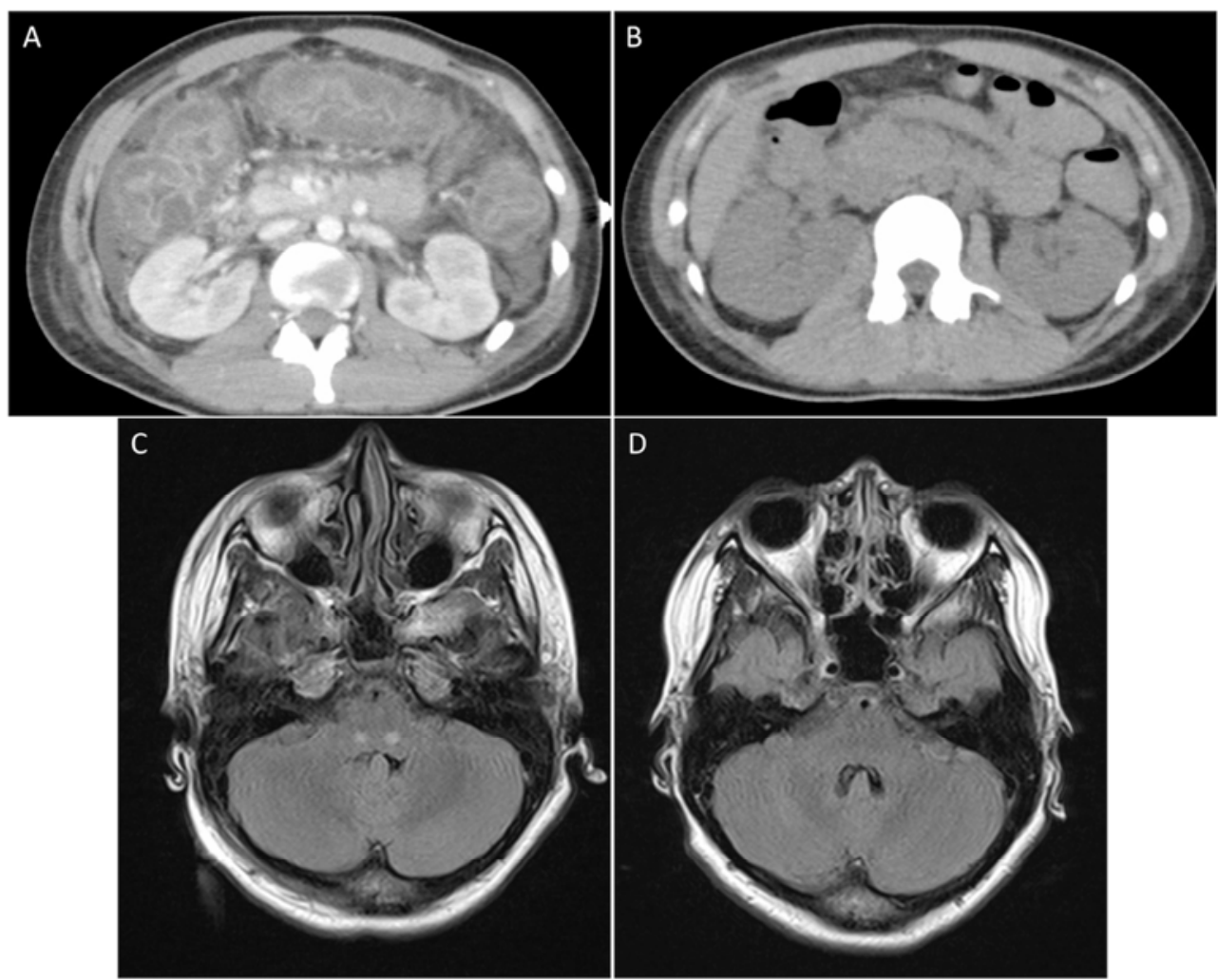

Figure 1. An abdominothoracic contrast-enhanced computed tomography and A head magnetic resonance image.

Legend: An abdominothoracic contrast-enhanced computed tomography image taken at hospital admission (A) showing pan-colonic membranous edema and ascites. These findings were almost improved by Day 14 of hospitalization as shown in the plain computed tomography image on the right (B). A head magnetic resonance image (MRI) taken at hospital admission showing a high-intensity area in the bilateral medial lemniscus of the pons (C). The abnormal lesions had disappeared in an MRI taken at Day 17 of hospitalization (D).

\subsection{Clinical Course After Admission}

The clinical course and details of the treatment after admission are summarized in Figure 2.



Figure 2. Levels of platelet counts and serum creatinine during hospitalization. 
Legend: The blue line shows the changes in platelet count after admission, while the orange line shows the changes in serum creatinine after admission. Human recombinant thrombomodulin was administered from Day 1 to 7 . Plasma exchange was performed on Day 3 and 4. High-dose methylprednisolone therapy ( $1000 \mathrm{mg} /$ day) was administered from Day 3 to 5 . Continuous hemodiafiltration was performed for acute renal failure from Day 1 to 8 . Intermittent hemodialysis was performed on Day 9 and 11 . Eculizumab was administered on Day 4, 11, 18, and 25. Levetiracetam for localized seizure was administered from Day 5. Carbamazepine was added from Day 14 and stopped at Day 32. The patient was discharged on Day 40 of hospitalization.

Abbreviations: PE, plasma exchange; hrTM, human recombinant thrombomodulin; mPSL, methyl prednisolone; CHDF, continuous hemodiafiltration; HD, hemodialysis; LEV, levetiracetam; CBZ, carbamazepine.

Because results of the direct Shiga-toxin detection tests conducted at the first hospital and repeated after admission were negative, we based our initial treatment on a presumed diagnosis of acute bacterial colitis caused by an infectious organism other than enterohemorrhagic E. coli. As sepsis and septic disseminated intravascular coagulation (DIC) were noted, the patient was managed with fasting and replacement fluids, continuous hemodiafiltration for oliguria, and recombinant thrombomodulin for septic DIC. The patient's clinical symptoms remained poor despite these treatments, with a worsening of renal function. On Day 3 post-admission (Day 7 after the onset of symptoms), the patient experienced systemic clonic convulsions. Although the convulsions ceased, altered consciousness persisted, and we performed tracheal intubation to secure the airway and placed the patient on a respirator. Ocular bobbing with abnormal deep tendon reflexes of the extremities and a Babinski sign bilaterally were observed. Extensive cortical disorder and brain stem disturbance were suspected and, accordingly, a head CT and magnetic resonance (MR) imaging were performed to ascertain the cause of the convulsive seizures. Although the head CT showed no clear abnormalities, the head MRI revealed intense signals near the medial lemniscus on both sides of the pons on the T2-FLAIR image sequence. (Figure 1C). The diffusion-weighted image and apparent diffusion coefficient map of the same site also revealed intense signals. Based on these findings, the patient was diagnosed with acute encephalopathy associated with HUS.

Due to suspicion of vascular edema, based on the MR imaging findings, steroid pulse therapy and plasma exchange were initiated, and treatment with levetiracetam was started as an anticonvulsant. Plasma exchange was also performed on Day 4 post-admission. Despite these interventions, the improvement in clinical symptoms, including test data, was poor. On Day 4, we received the in-depth report of the fecal culture test performed at the first clinic, which revealed enterohemorrhagic E. coli $\mathrm{O} 157$ and Shiga-toxin type 2, leading to the definitive diagnosis of STEC-HUS. Based on the results of the tests performed since admission, collagen and autoimmune diseases were ruled out and secondary HUS was not actively suspected.

Considering the severe complications experienced by the patient and the poor improvement in symptoms following treatment, we could not rule out the involvement of a complement-mediated pathology in the STEC-HUS pathology. Accordingly, we initiated eculizumab treatment, which is a terminal complement inhibitor, and ceased plasma exchange. After starting eculizumab, the platelet count increased on a daily basis and the serum creatinine level also improved. As a urine sample was obtained on Day 7 post-admission, continuous hemodiafiltration was terminated on Day 8. Intermittent hemodialysis was performed on Days 9 and 11, with renal replacement therapy being withdrawn thereafter and the hemodialysis tube removed on Day 11 . However, left-sided conjugate gaze palsy and upper and lower left limb tonic-clonic seizures reappeared on Day 14 . Diagnostic imaging revealed no abnormalities. However, convulsive waves originating from the right temporoparietal lobe were observed during electroencephalography. We considered this as a secondary generalization of a partial seizure and initiated carbamazepine therapy. No repeat convulsions occurred thereafter, and the patient's consciousness improved. MRI performed on Day 17 showed that the region of intense signal on both sides of the pons had disappeared (Figure 1D). Electroencephalography was repeated on Day 30. Moreover, because no clear abnormalities were observed, carbamazepine was discontinued on Day 32, and only levetiracetam was continued. The patient's general condition steadily improved and on Day 40, she was discharged without any permanent neurological or organ damage.

\section{Discussion}

STEC characteristically causes bloody stool with HUS identified as a complication of STEC infection in 6-9\% of cases [3]. Typically, clinical symptoms, such as bloody diarrhea and abdominal pain, manifest 3 or 4 days after STEC infection [6]. The prevalence rate of STEC-specific mortality has been estimated at 1-2\% [7], with a higher risk of mortality among elderly individuals and those with HUS complication. The following tests are useful for the diagnosis of STEC infection: fecal culture; fecal Shiga-toxin detection, and anti-lipopolysaccharide-IgM antibody tests. HUS is characterized by three primary pathologies, namely acute renal dysfunction, microvascular hemolytic anemia, and consumption thrombotic thrombocytopenia. Typically, HUS manifests 5-10 days after the onset of diarrhea [8]. Among patients with an STEC infection that progresses to HUS, 50\% of cases require hemocatharsis, with an overall mortality rate of 3-5\% [7]. Over the longer term of HUS, renal impairment, including hypertension and proteinuria occurs in 39\% of cases, with permanent neurological impairment, including convulsions, stroke, and coma occurring in $4 \%$ of cases $[9$, 
10]. Although studies have suggested that antimicrobial treatment of STEC infections increases the risk of HUS, a meta-analysis did not identify a significant difference in HUS risk after antimicrobial therapy [11].

STEC-HUS is a differential diagnosis of TMA. In addition to STEC-HUS, TMA includes thrombotic thrombocytopenic purpura (activation level of a disintegrin-like and metalloproteinase with thrombospondin type 1 motifs 13 (ADAMTS13) significantly falls below 10\%), autoimmune/collagen diseases and invasive pneumococcal infection, pregnancy-related and drug-induced TMA, and complement-related HUS (aHUS). The Pediatric HUS Care Guideline of the Japanese Society of Nephrology in 2000 recommends the use of supportive treatment for STEC-HUS, which includes fluid replacement therapy, nutritional management by central venous hyperalimentation, blood transfusion, blood pressure management, and treatment for acute renal disorder, encephalopathy, and DIC. For patients who develop encephalopathy, plasma exchange and steroid pulse therapy have been empirically administered $[12,13]$.

Eculizumab is a complement (C5) monoclonal antibody preparation that selectively binds to the complement $\mathrm{C} 5$ to suppress its decomposition to $\mathrm{C} 5 \mathrm{a}$ and $\mathrm{C} 5 \mathrm{~b}$, as well as suppressing the production of $\mathrm{C} 5 \mathrm{a}$ and MAC. This results in the inhibition of the complement activation pathway. Eculizumab was originally approved in 2007 in Europe and the United States and in 2010 in Japan for the treatment of PNH. In 2013, the use of eculizumab in Japan was extended to the treatment of aHUS. aHUS can be classified into loss-of-function mutation in the complement regulatory factor or a gain-of-function mutation in the complement activation factor. In either case, excessive activation of the second of the complement activation pathways may activate vascular endothelial cells and platelets' surface antigens to bring about complement-mediated TMA. If aHUS is strongly suspected, based on measured levels $\mathrm{C} 3$ and $\mathrm{C} 4$ complements in the blood, tests for known causative genetic mutations (CFH, CFB, CFI, C3, CD46, THBD, DGKE, [PLG]) should be performed, with identification of the presence/absence of the anti-H-factor antibody being analyzed for diagnosis. Because known genetic mutations are absent in about $40 \%$ of patients, aHUS cannot be ruled out even if genetic mutations are unnoted [14, 15]. In a Japanese study, eculizumab was used in 10 cases of pediatric aHUS with and without aHUS-related genetic mutation, and the platelet count improved after about 1 week of eculizumab therapy [16]. These findings indicate that for cases in which eculizumab is found to be effective, aHUS due to complement system abnormality should be considered, even if a causative genetic mutation is unidentifiable. The patient in our case report tested negatively for anti-H-antibody, without a known genetic mutation.

According to the 2013 diagnostic criteria for aHUS, eculizumab is an effective treatment for aHUS, which targets the abnormalities in complement regulation. However, the use of eculizumab for STEC-HUS and for secondary TMA is not currently recommended. However, Lapeyraque et al. suggested that eculizumab treatment should be considered for the treatment of severe cases of STEC-HUS that have progressed to encephalopathy and when there is a poor response to plasma exchange therapy [5].

According to Mizuguchi et al. [17], STEC-HUS associated encephalopathy can result from the following mechanisms: increased vascular permeability due to the collapse of the cerebrovascular barrier in vessels where the Shiga-toxin has been deposited, direct disorders caused by the deposition of Shiga-toxin on nerve tissues, and cerebrovascular accidents due to cerebrovascular wall thickening and thrombus formation. These mechanisms result in diffuse cerebral edema due to protein leakage around the affected blood vessel and selective damage to Purkinje cells [18].

Fitzpatrick et al. reported a high relationship between the level of interleukin- 8 and chemotactic activation of neutrophils (and the levels of granulocyte esterase released from neutrophils) in patients with severe STEC-HUS [19]. The increased abnormal activation of the complement activation pathway due to these cytokines is considered to be the causative pathway for complement-mediated TMA [20]. Furthermore, Fang et al. identified genetic mutations in autologous cell membrane surface protein in patients with lethal forms of STEC-HUS infection [21].

Considering the known pathophysiological pathways of STEC-HUS, inhibition of the complementary regulatory pathway using eculizumab is likely to be effective in inhibition the biological response to STEC infections. A prospective study by Legendre et al. [4] indicated that the early intervention using eculizumab can be effective in suppressing complement-mediated TMA, reducing the need to manage TMA-related events and, notably, improving platelet count and renal dysfunction.

\section{Conclusion}

Based on our experience, we propose that eculizumab should be considered for the treatment of patients with STEC-HUS who show poor clinical improvement and test values. In particular, eculizumab might be effective for cases of severe STEC-HUS that progress to encephalopathy. The effectiveness of eculizumab in these case would be mediated through an inhibition of the abnormal activation of the complement activation pathway, which is enhanced by the STEC infection.

\section{References}

[1] O’Brien, A. O., T. A. Lively TA, M. E. Chen, S. W. Rothman, and S. B. Formal. (1983) Escherichia coli O157:H7 strains associated with haemorrhagic colitis in the United States produce a Shigella dysenteriae 1 (SHIGA) like cytotoxin. The Lancet 1, 702.

[2] Frank, C., D. Werber, J. P. Cramer, M. Askar, M. Faber, M. an der Heiden, et al. (2011) Epidemic profile of Shiga-toxin-producing Escherichia coli O104:H4 outbreak in Germany. The New England Journal of Medicine 365, 1763. 
[3] Tarr, P. I., C. A. Gordon, and W. L. Chandler. (2005) Shiga-toxin-producing Escherichia coli and haemolytic uraemic syndrome. The Lancet 365, 1073-1086.

[4] Legendre, C. M., C. Licht, P, Muus, L. A. Greenbaum, S. Babu, et al. (2013) Terminal complement inhibitor eculizumab in atypical hemolytic-uremic syndrome. The New England Journal of Medicine 368, 2169-2181.

[5] Lapeyraque, A. L., M. Malina, V. Fremeaux-Bacchi, T. Boppel, M. Kirschfink, et al. (2011) Eculizumab in severe Shiga-toxin-associated HUS. The New England Journal of Medicine 364, 2561-2563.

[6] Slutsker, L., A. A. Ries, K. D. Greene, J. G. Wells, L. Hutwagner, et al. (1997) Escherichia coli O157:H7 diarrhea in the United States: clinical and epidemiologic features. Annals of Internal Medicine 126, 505-513.

[7] Boyce, T. G., D. L. Swerdlow, and P. M. Griffin. (1995) Escherichia coli O157:H7 and the hemolytic-uremic syndrome. The New England Journal of Medicine 333, 364-368.

[8] Su, C., and L. J. Brandt. (1995) Escherichia coli O157:H7 infection in humans. Annals of Internal Medicine 123, 698-714.

[9] Siegler, R. L., M. K. Milligan, T. H. Burningham, R. D. Christofferson, S. Y. Chang, et al. (1991) Long-term outcome and prognostic indicators in the hemolytic-uremic syndrome. Journal of Pediatrics 118, 195-200.

[10] Rosales, A., J. Hofer, L. B. Zimmerhackl, T. C. Jungraithmayr, M. Riedl, et al. (2012) Need for long-term follow-up in enterohemorrhagic Escherichia coli-associated hemolytic uremic syndrome due to late-emerging sequelae. Clinical Infectious Diseases 54, 1413-1421.

[11] Freedman, S. B., J. Xie, M. S. Neufeld, W. L. Hamilton, L. Hartling, et al. (2016) Shiga toxin-producing Escherichia coli infection, antibiotics, and risk of developing hemolytic uremic syndrome: A meta-analysis. Clinical Infectious Diseases 62, 1251-1258.

[12] Nathanson, S., T. Kwon, M. Elmaleh, M. Charbit, E. A. Launay, et al. (2011) Acute neurological involvement in diarrhea-associated hemolytic uremic syndrome. Clinical Journal of the American Society of Nephrology 5, 1218-1228.
[13] Fujii J, Y. Kinoshita, A. Matsukawa, S. Y. Villanueva, T. Yutsudo, et al. (2009) Successful steroid pulse therapy for brain lesion caused by Shiga toxin 2 in rabbits. Microbial Pathogenesis 46, 179-184.

[14] Roumenina, L. T., R. Roquigny, C. Blanc, N. Poulain, S. Ngo, et al. (2014) Functional evaluation of factor $\mathrm{H}$ genetic and acquired abnormalities: application for atypical hemolytic uremic syndrome (aHUS). Methods in Molecular Biology $1100,237-247$.

[15] Noris, M., J. Caprioli, E. Bresin, C. Mossali, G. Pianetti, et al. (2010) Relative role of genetic complement abnormalities in sporadic and familial aHUS and their impact on clinical phenotype. Clinical Journal of the American Society of Nephrology 5, 1844-1859.

[16] Ito, N., H. Hataya, K. Saida, Y. Amano, Y. Hidaka, et al. (2016) Efficacy and safety of eculizumab in childhood atypical hemolytic uremic syndrome in Japan. Clinical and Experimental Nephrology 20, 265-272.

[17] Mizuguchi, M., S. Tanaka, I. Fujii, H. Tanizawa, Y. Suzuki, et al. (1996) Neuronal and vascular pathology produced by verocytotoxin 2 in the rabbit central nervous system. Acta Neuropathologica 91, 254-262.

[18] Fujii, J., T. Kita, S. Yoshida, T. Takeda, H. Hobayashi, et al. (1994) Direct evidence of neuron impairment by oral infection with verotoxin-producing Escherichia coli O157: H-in mitomycin-treated mice. Infection and Immunity 62, 3447-3453.

[19] Fitzpatrik, M. M., V. Shah, R. S. Trompeter, M. J. Dillon, T. M. Barratt. (1992) Interleukin-8 and polymorphoneutrophil leucocyte activation in hemolytic uremic syndrome of childhood. Kidney International 142, 951-956.

[20] Thurman, J. M., R. Marians, W. Emlen, S. Wood, C. Smith, et al. (2009) Alternative pathway of complement in children with diarrhea-associated hemolytic uremic syndrome. Clinical Journal of the American Society of Nephrology 4, 1920-1924.

[21] Fang, C. J., V. Frémeaux-Bacchi, M. K. Liszewski, G. Pianetti, M. Noris, et al. (2008) Membrane cofactor protein mutations in atypical hemolytic uremic syndrome (aHUS), fatal Stx-HUS, C3 glomerulonephritis, and the HELLP syndrome. Blood 111, 624-632. 